\title{
Desenvolvimento e avaliação de jogos para a inclusão digital de pessoas idosas
}

\author{
Johnnie M. Giacomelli ${ }^{1}$, Aline V. de Mello ${ }^{1}$, \\ Jean F. P. Cheiran ${ }^{1}$, Miguel J. Z. da Costa Junior ${ }^{1}$ \\ ${ }^{1}$ Universidade Federal do Pampa (UNIPAMPA) - campus Alegrete \\ 97546-550 - Alegrete - RS - Brasil \\ \{johnniegiacomelli,mzinelli95\}@gmail.com, \\ \{jeancheiran, alinemello\}@unipampa.edu.br
}

\begin{abstract}
Since the older adult population is rising year by year, the policies of digital inclusion for older people are essential to avoid technological and social isolation. Also, as many elders return to work to complement their pensions, they need to learn how to use computers in many situations. The goal of this research is to develop web browser games for mouse and keyboard training and to assess their effectiveness in improving the skill of older people after short training. Our key findings include that a few training sessions (1) contribute little to improve the movement and click precision with the mouse, (2) were enough to increase significantly the time performance with the mouse, and (3) increased substantially the amount of typed words in a time frame.
\end{abstract}

Resumo. Uma vez que a população idosa está crescendo ano após ano, as políticas de inclusão digital para idosos são essenciais para evitar isolamento tecnológico e social. Ainda, conforme muitos idosos retornam ao trabalho para complementar suas aposentadorias, eles precisam aprender a usar computadores em muitas situações. O objetivo dessa pesquisa é desenvolver jogos para navegadores para treinamento de mouse e teclado e aferir sua efetividade na melhoria de habilidade dos idosos depois de um treinamento curto. Nossas principais descobertas incluem que poucas sessões de treino (1) contribuem pouco para melhorar a precisão de movimento e clique com o mouse, (2) foram suficientes para aumentar significativamente a velocidade com o mouse, $e$ (3) aumentaram substancialmente a quantidade de palavras digitadas em um intervalo de tempo.

\section{Introdução}

De acordo com a Organização das Nações Unidas (ONU), a população de idosos ${ }^{1}$ está aumentando em praticamente todos os países [ONU 2018] devido à melhora da qualidade de vida, aos avanços da medicina e às novas tecnologias [ONU 2018]. Entretanto, o potencial dos idosos ainda é esquecido ou negligenciado no âmbito social e de trabalho [Kachar 2003], fato agravado pelo declínio de funções cognitivas e motoras, caracterizando assim um antigo estereótipo de que o idoso é incapaz de trabalhar em suas funções anteriores [Kachar 2003].

\footnotetext{
${ }^{1}$ Idosos são caracterizados no Brasil como pessoas acima de 60 anos [Brasil 2003].
} 
Ainda assim, há idosos que respondem por cerca de metade da fonte de renda de muitas famílias [Fiocruz 2020] e alguns têm a necessidade de retornar ao mercado de trabalho, esbarrando em problemas ao usar novas Tecnologias de Informação e Comunicação (TIC), como programas de computador e aplicativos de smartphones. Além disso, com a pandemia do novo coronavírus, esses idosos que são o maior grupo de risco da Covid-19 devem permanecer em distanciamento social, transferindo seu trabalho para a modalidade home office [Fiocruz 2020] e fazendo essencial o uso das TIC [Vieira 2011]. Isso faz com que muitas atividades sejam desenvolvidas exclusivamente pelo computador, sendo indispensável o domínio de mouse e teclado.

Problemas na coordenação motora e na motricidade fina, acarretados muitas vezes pela falta de exercício de músculos das mãos, podem trazer dificuldades no uso desses periféricos, que muitas vezes são vistos como os grandes vilões para o aprendizado [Kachar 2003, Vieira 2011]. Neste sentido, os jogos podem ser utilizados para desenvolver habilidades de forma mais lúdica [Aldrich 2005].

Considerando que é significativo avaliar a efetividade do treinamento de idosos por meios lúdicos para aprimorar suas habilidades com o computador, colocamos as seguintes questões de pesquisa para nortear o desenvolvimento deste trabalho: (1) Idosos realizam movimentação mais precisa com o mouse após o treinamento com jogos? (2) Idosos realizam cliques mais precisos com o mouse após o treinamento? (3) Idosos realizam tarefas mais rapidamente com o mouse após o treinamento? (4) Idosos conseguem digitar palavras mais rapidamente usando o teclado após o treinamento?

Assim, o objetivo deste artigo é desenvolver jogos para web e avaliar sua efetividade enquanto ferramentas que visam aperfeiçoar a utilização do mouse e do teclado em um experimento de treinamento curto com pessoas idosas. Para isso, foram desenvolvidos (1) sete jogos com temática gaúcha para treinamento do uso de mouse e teclado, (2) testes para avaliação de melhoria visomotora das habilidades treinadas pelos jogos, e (3) um estudo de caso na forma de um treinamento presencial para idosos.

\section{Trabalhos Relacionados}

Nesta seção são apresentados outros trabalhos recentes em que os autores desenvolveram um jogo sério para idosos e realizaram avaliação com o público-alvo. A Tabela 1 apresenta a referência para cada trabalho, o nome e o objetivo do jogo, e o número de idosos que participaram do processo de avaliação.

Os jogos Music Therapy, Safari, Cow Milking e Motriz têm como objetivo desenvolver aspectos cognitivos em idosos. Os jogos Skiing e WipeOut foram desenvolvidos para motivar idosos a realizar exercícios físicos regulares, sendo que o jogo WipeOut é focado em exercícios de reabilitação. O jogo Motriz, além de trabalhar aspectos cognitivos, desenvolve funções motoras, estimulando idosos a utilizarem o mouse de forma mais rápida, precisa e correta.

A maioria dos trabalhos usou estatística inferencial para avaliar os resultados obtidos, exceto o jogo Motriz. Os autores do jogo Motriz concluíram que os idosos tiveram dificuldades em realizar as atividades do jogo, porém indicaram que os idosos apresentaram uma melhoria em relação ao passado sem apresentar as evidências dessa melhora.

No jogo Music Therapy, o desempenho inicial e final de cada participante foi 
IX Congresso Brasileiro de Informática na Educação (CBIE 2020)

Anais do XXXI Simpósio Brasileiro de Informática na Educação (SBIE 2020)

Tabela 1. Trabalhos relacionados ao desenvolvimento de jogos para idosos

\begin{tabular}{|l|l|l|r|}
\hline \multicolumn{1}{|c|}{ Autores } & \multicolumn{1}{c|}{ Jogo } & \multicolumn{1}{c|}{ Objetivo } & No $^{\mathbf{0}}$ Idosos \\
\hline [Cheng et al. 2019] & Music Therapy & Cognição & 5 \\
\hline [Niksirat et al. 2017] & Safari & Cognição & 20 \\
\hline [Neto et al. 2016] & Cow Milking & Cognição & 106 \\
\hline [Bonnechère et al. 2016] & WipeOut & Exercícios Físicos & 22 \\
\hline [Pyae et al. 2016] & Skiing & Exercícios Físicos & 24 \\
\hline [Farias et al. 2015] & Motriz & Cognição e Motricidade & 20 \\
\hline
\end{tabular}

comparado usando o teste $t$ de Student pareado. Dos 7 participantes do processo de avaliação, 4 aumentaram significativamente suas habilidades em jogar, 2 mantiveram seu desempenho desde o inicio, e 1 apresentou desempenho inferior na fase final. No jogo Skiing, os resultados demonstraram que existe uma correlação significativa entre o afeto positivo durante o jogo e a experiência positiva pós-jogo. Adicionalmente, os idosos relataram que a usabilidade do jogo foi perceptivelmente positiva.

No jogo Safari, o teste ANOVA para medidas repetidas foi usado. Dentre as conclusões destacaram-se: idosos preferem o ajuste de dificuldade manual ao dinâmico e o recurso Kinect ao GamePad. No jogo Cow Milking, a correlação de Spearman $(r)$ foi usada para relacionar os resultados obtidos pelos idosos na avaliação cognitiva e no jogo. O jogo mostrou-se mais adequado aos idosos com comprometimento cognitivo. Já no jogo WipeOut, o coeficiente de correlação de Pearson foi usado para estudar a convergência da avaliação clínica e da pontuação obtida no jogo. Os autores concluíram que a plataforma vibratória possui melhor resultado do que o Kinect. No entanto, do ponto de vista da reabilitação, ambos devem ser usados para combinar diferentes exercícios de reabilitação.

\section{Jogos Desenvolvidos}

Sete jogos com a temática Gaúcha foram desenvolvidos (Figura 1) e são descritos a seguir.

1. Pulando cupinzeiros: o jogador joga com o cavalo e deve clicar para fazer com que ele salte sobre os cupinzeiros que vem em sua direção.

2. Pegando balões: o jogador joga com o pássaro quero-quero e o seu objetivo é pegar os balões na tela e depois ir até a bandeira do Rio Grande do Sul.

3. Encontrando o gaúcho: o jogador deve clicar no local em que o gaúcho aparece.

4. Memória gaúcha: é um jogo de memória clássico que usa imagens gauchescas.

5. Entregando chimarrão: o jogador tem o papel da mão do Gaúcho que deve pegar o chimarrão e levá-lo até as mãos da Prenda.

6. Pegando ovos: o jogador deve pressionar a tecla correspondente a letra mostrada dentro do ovo antes que ele quebre no chão.

7. Laçando vaca: o jogador joga com o gaúcho que laça a vaca quando as teclas necessárias para formar a letra com acento mostrada são pressionadas.

Cada jogo treina funções específicas de mouse ou do teclado, conforme a Tabela 2.

A funcionalidade de clicar no botão esquerdo do mouse sem a necessidade de precisão fina é treinada pelo jogo Pulando cupinzeiros (Figura 1a). Já a precisão fina é 


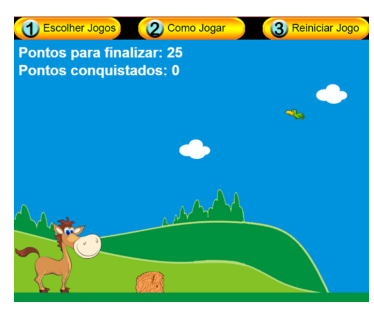

(a) Pulando cupinzeiros

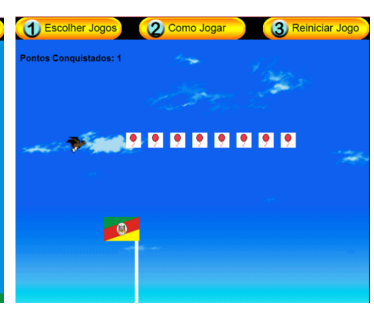

(b) Pegando

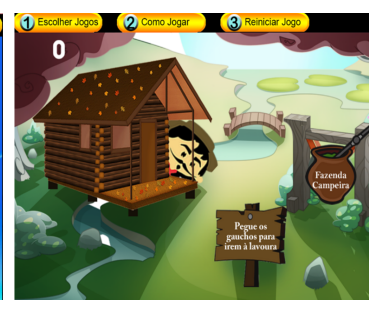

(c) Encontrando

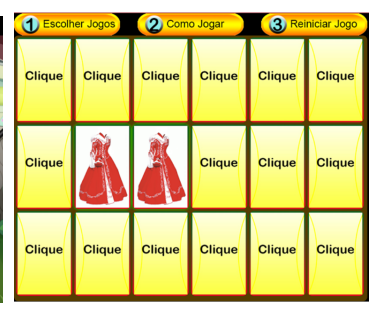

(d) Memória

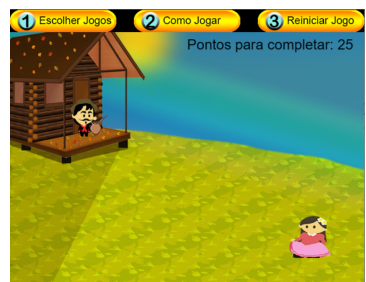

(e) Entregando

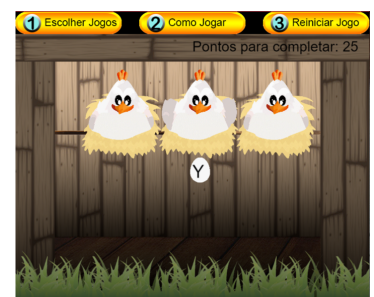

(f) Pegando o gaúcho

chimarrão

ovos

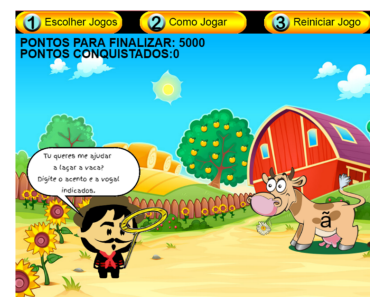

(g) Laçando
a vaca

Figura 1. Capturas de telas dos jogos desenvolvidos

treinada pelos jogos Memória gaúcha (Figura 1d), Entregando chimarrão (Figura 1e) e Encontrando o gaúcho (Figura 1c). Nesses jogos, o jogador deve clicar em uma imagem que possui uma localização específica na tela.

A movimentação livre do mouse é treinada nos jogos Pegando balões (Figura 1b), Memória gaúcha (Figura 1d) e Encontrando o gaúcho (Figura 1c), enquanto a movimentação drag and drop é treinada no jogo Entregando o chimarrão (Figura 1e), em que o jogador deve clicar no botão esquerdo do mouse quando o cursor está em cima da imagem do chimarrão e mantê-lo pressionado durante o trajeto até as mãos da Prenda.

Tabela 2. Jogos e suas funcionalidades de treinamento

\begin{tabular}{|l|c|c|c|c|c|c|}
\hline \multirow{2}{*}{ Nome } & \multicolumn{4}{c|}{ Mouse } & \multicolumn{2}{c|}{ Teclado } \\
\cline { 2 - 7 } & \multicolumn{2}{|c|}{ Clique } & \multicolumn{2}{c|}{ Movimentação } & \multirow{2}{*}{ Letra } & \multirow{2}{*}{ Acento } \\
\cline { 2 - 7 } & Livre & Preciso & Livre & Drag'n'drop & & \\
\hline \hline Pegando balões & & & $\mathrm{x}$ & & & \\
\hline Pulando cupinzeiros & $\mathrm{x}$ & & & & & \\
\hline Memória gaúcha & & $\mathrm{x}$ & $\mathrm{x}$ & & & \\
\hline Entregando chimarrão & & $\mathrm{x}$ & & $\mathrm{x}$ & & \\
\hline Encontrando o gaúcho & & $\mathrm{x}$ & $\mathrm{x}$ & & & \\
\hline Pegando ovos & & & & & $\mathrm{x}$ & \\
\hline Laçando vaca & & & & & $\mathrm{x}$ & $\mathrm{x}$ \\
\hline
\end{tabular}

\section{Metodologia}

\subsection{Criação e avaliação dos jogos}

Elementos, cenários e atividades tradicionais do Pampa Gaúcho compuseram a estética e a narrativa dos jogos desenvolvidos. A adoção de um contexto familiar favoreceu o 
envolvimento dos idosos e desviou a atenção das mecânicas de jogo.

O design da estética e das mecânicas de cada jogo foi realizado por meio de prototipação em papel. Ainda, uma inspeção heurística aplicada sobre os protótipos com mecânicas simuladas e um teste de usabilidade com seis usuários realizado nas versões preliminares dos jogos são detalhados em um estudo anterior [Giacomelli et al. 2018].

Os jogos foram desenvolvidos usando HTML5, CSS3 e JavaScript, e a compatibilidade com os navegadores Google Chrome, Mozilla Firefox e Microsoft Edge foi garantida. O framework Phaser versão 3.0 foi utilizado em alguns dos jogos. Os assets de imagem e som foram obtidos em bibliotecas de uso livre ou criados pela equipe.

\subsection{Participantes}

Participaram do experimento 13 pessoas com idades entre 61 e 74 anos $\left(\bar{x}=67.85, s_{x}=\right.$ 4.059). Nesse grupo, 2 participantes usam computador com mouse e teclado diariamente, 1 participante usa às vezes, 6 participantes usam raramente, e 4 participantes nunca usaram mouse ou teclado antes desse experimento.

\subsection{Protocolo experimental}

O experimento foi realizado ao longo de cinco dias. No primeiro dia, os participantes foram apresentados à pesquisa, preencheram o Termo de Consentimento Livre e Esclarecido (TCLE), um questionário de perfil e o Short Portable Mental Status Questionnaire ${ }^{2}$ (SPMSQ) [Pfeiffer 1975]. Posteriormente, eles realizaram o teste de desempenho com mouse e teclado (pré-treino) e participaram da primeira sessão de treino com os jogos. Nos próximos três dias, os participantes realizaram somente a sessão de treino com os jogos. No quinto e último dia, os participantes realizaram somente o teste de desempenho com mouse e teclado (pós-treino).

O experimento foi realizado em um laboratório de informática da UNIPAMPA, usando computadores com Windows 10 e o navegador Google Chrome. Cada sessão de treino com os jogos foi acompanhada por monitores e teve duração de 30 minutos e a ordem de aplicação dos jogos foi a mesma da Figura 1. A quantidade de tempo em cada um dos jogos variou, porém todos os jogos foram jogados ao menos uma vez em cada sessão.

Embora o experimento completo tenha sido realizado em 5 dias. a frequência dos idosos variou: 2 idosos participaram de 2 dias, 4 participaram de 3 dias, 4 participaram de 4 dias, e 3 participaram de todos os 5 dias.

\subsection{Testes de desempenho com mouse e teclado}

O desempenho dos idosos foi aferido por meio da habilidade de percurso com o mouse em espaços delimitados com o botão esquerdo pressionado (Figuras 2a, 2b, 2c e 2d), da habilidade de realizar cliques únicos sem movimentação do mouse (Figura 2e), e da habilidade de digitar palavras visualizadas na tela (Figura 2f).

Nas avaliações de desempenho com mouse, o software Microsoft Paint foi usado para permitir que o idoso desenhasse linhas contínuas das áreas de partida mais à esquerda

\footnotetext{
${ }^{2}$ O SPMSQ [Pfeiffer 1975] foi traduzido para português brasileiro e usado para aferir a ausência de comprometimento intelectual dos idosos. Todos os participantes se mostraram dentro dos parâmetros.
} 


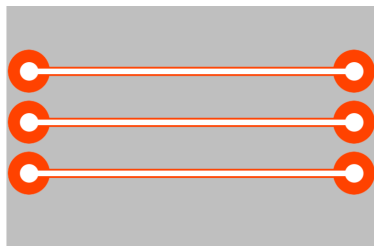

(a) Horizontal

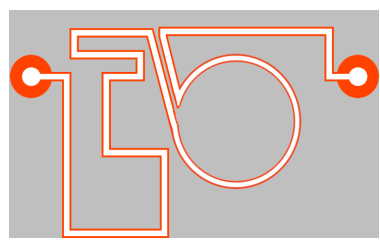

(d) Misto

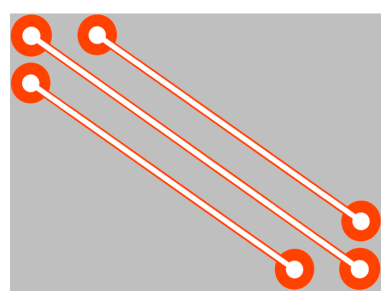

(b) Diagonal

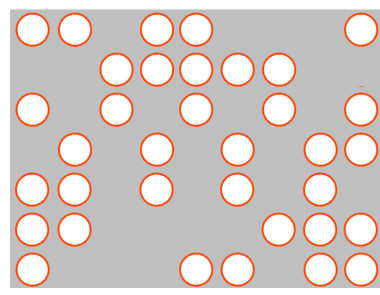

(e) Clique

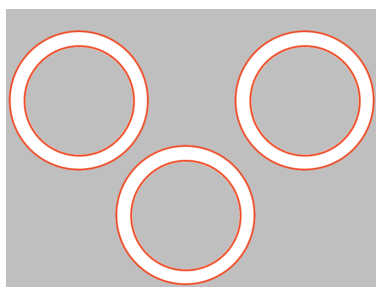

(c) Circular

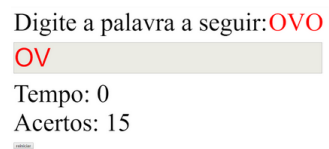

(f) Digitação

Figura 2. Teste de desempenho com mouse e teclado em relação a movimentos horizontais, diagonais, circulares e mistos, cliques, e digitação.

até aquelas mais à direita (Figuras $2 \mathrm{a}, 2 \mathrm{~b}$ e $2 \mathrm{~d}$ ); desenhasse linhas contínuas de um ponto qualquer do círculo até completar a circunferência (Figura 2c); e desenhasse pontos na área interna de cada círculo (Figura 2e). Os monitores garantiram que todos os participantes realizassem esses testes utilizando da ferramenta "Lápis" com tamanho de 1 pixel e cor preta. Os monitores também reforçaram que as linhas e pontos deveriam se manter dentro das áreas brancas e cronometraram os tempos para concluir as tarefas.

$\mathrm{Na}$ avaliação de desempenho com teclado, um sistema desenvolvido usando HTML5 e JavaScript foi adotado para que os idosos fossem apresentados a palavras na tela (uma por vez) e as digitassem no campo de texto disponível (Figura 2f). O idoso deveria digitar quantas palavras conseguisse no tempo de 100 segundos. A lista de palavras seguiu a mesma ordem para todos os participantes (incluindo no teste pós-treino) e seu limite não foi atingido por nenhum participante.

\subsection{Questões éticas}

Os procedimentos experimentais foram elaborados em consonância com recomendações do Comitê de Ética em Pesquisa da UNIPAMPA. Todos os participantes foram informados dos detalhes do experimento, preencheram e assinaram o TCLE, e ficaram com cópias do Termo de Confidencialidade (TC) e do TCLE assinados pelos pesquisadores.

\section{Resultados}

O software IBM SPSS Statistics 26 foi usado para estatística descritiva e inferencial.

\subsection{Avaliação de desempenho com mouse}

Testes Shapiro-Wilk para aferição de normalidade aplicados sobre as amostras de erros e tempos da Tabela 3 revelam dados com distribuições heterogêneas com pouco mais da

\footnotetext{
${ }^{3}$ Os tipos de erro avaliados nos testes na Figura 2 incluíram avançar sobre ou encostar na linha delimitadora laranja (tocar), cruzar completamente a linha delimitadora laranja (cruzar), liberar o botão do mouse e pressioná-lo novamente (soltar), e preencher mais que um pixel na tentativa de fazer um ponto (não ponto).
} 
IX Congresso Brasileiro de Informática na Educação (CBIE 2020)

Anais do XXXI Simpósio Brasileiro de Informática na Educação (SBIE 2020)

Tabela 3. Estatística descritiva para erros e tempos (em segundos) nos testes de movimento e cliques de mouse.

\begin{tabular}{|c|c|c|c|c|c|c|c|}
\hline \multirow{2}{*}{ Teste } & \multirow{2}{*}{ Momento } & \multirow{2}{*}{$\begin{array}{c}\text { Tipo de } \\
\text { Erro }^{3}\end{array}$} & \multirow{2}{*}{$\mathbf{N}$} & \multicolumn{2}{|c|}{$\mathbf{N}^{\circ}$ Erros } & \multicolumn{2}{|c|}{ Tempos } \\
\hline & & & & $\bar{x}$ & $s_{x}$ & $\bar{x}$ & $s_{x}$ \\
\hline \multirow{6}{*}{$\begin{array}{c}\text { movimento } \\
\text { horizontal }\end{array}$} & \multirow{3}{*}{ pré-treino } & tocar & 13 & 8.31 & 3.497 & \multirow{3}{*}{124.31} & \multirow{3}{*}{82.12} \\
\hline & & cruzar & 13 & 10.08 & 9.996 & & \\
\hline & & soltar & 13 & 8.69 & 10.020 & & \\
\hline & \multirow{3}{*}{ pós-treino } & tocar & 13 & 7.23 & 4.494 & \multirow{3}{*}{90.85} & \multirow{3}{*}{69.37} \\
\hline & & cruzar & 13 & 3.77 & 4.323 & & \\
\hline & & soltar & 13 & 1.46 & 1.198 & & \\
\hline \multirow{6}{*}{$\begin{array}{c}\text { movimento } \\
\text { diagonal }\end{array}$} & \multirow{3}{*}{ pré-treino } & tocar & 13 & 17.69 & 6.434 & \multirow{3}{*}{93.31} & \multirow{3}{*}{42.78} \\
\hline & & cruzar & 13 & 7.85 & 4.705 & & \\
\hline & & soltar & 13 & 1.92 & 2.216 & & \\
\hline & \multirow{3}{*}{ pós-treino } & tocar & 13 & 15.00 & 3.606 & \multirow{3}{*}{69.08} & \multirow{3}{*}{29.05} \\
\hline & & cruzar & 13 & 7.62 & 6.358 & & \\
\hline & & soltar & 13 & .85 & 1.519 & & \\
\hline \multirow{6}{*}{$\begin{array}{l}\text { movimento } \\
\text { circular }\end{array}$} & \multirow{3}{*}{ pré-treino } & tocar & 13 & 6.92 & 4.406 & \multirow{3}{*}{121.77} & \multirow{3}{*}{74.09} \\
\hline & & cruzar & 13 & 10.08 & 13.338 & & \\
\hline & & soltar & 13 & 3.00 & 2.646 & & \\
\hline & \multirow{3}{*}{ pós-treino } & tocar & 13 & 4.92 & 3.353 & \multirow{3}{*}{73.46} & \multirow{3}{*}{28.47} \\
\hline & & cruzar & 13 & 2.92 & 3.883 & & \\
\hline & & soltar & 13 & 1.69 & 1.932 & & \\
\hline \multirow{6}{*}{$\begin{array}{l}\text { movimentos } \\
\text { mistos }\end{array}$} & \multirow{3}{*}{ pré-treino } & tocar & 13 & 15.77 & 5.630 & \multirow{3}{*}{141.62} & \multirow{3}{*}{79.35} \\
\hline & & cruzar & 13 & 17.31 & 13.487 & & \\
\hline & & soltar & 13 & 3.31 & 4.733 & & \\
\hline & & tocar & 13 & 16.62 & 7.709 & & \\
\hline & pós-treino & cruzar & 13 & 11.31 & 7.532 & 105.62 & 44.84 \\
\hline & & soltar & 13 & 2.54 & 4.235 & & \\
\hline & pré-treino & não ponto & 13 & 18.00 & 10.638 & 117.62 & 53.36 \\
\hline es & pós-treino & não ponto & 13 & 17.62 & 8.694 & 89.54 & 36.49 \\
\hline
\end{tabular}

metade das amostras apresentando distribuição normal ( $p$-valor $>.05$ ). Dessa forma, buscando manter uma postura conservadora, aplicamos testes não-paramétricos de postos sinalizados de Wilcoxon para todas as comparações em uma perspectiva within-subjects.

A Tabela 4 apresenta o tamanho da amostra de cada par pré-treino e pós-treino $(\mathrm{N}-\mathrm{N})$, o escore $\mathrm{Z}(Z)$, a significância bicaudal ( $p$-valor) e a correlação que representa o tamanho de efeito $(r)$. Nessa tabela, constatamos que houve redução significativa na quantidade de erros em três casos: cruzar completamente a linha delimitadora e soltar o botão do mouse na realização de movimentos horizontais, e cruzar completamente a linha delimitadora na realização de movimentos circulares. Observamos também que o tamanho de efeito [Pallant 2016] para essas diferenças é médio $(r>.3)$ ou grande $(r>.5)$, corroborando uma melhora expressiva. Ainda, podemos identificar aumento significativo na velocidade dos participantes idosos para concluir as tarefas em todos os casos. O tamanho de efeito permanece médio ou grande para todos os testes. 
IX Congresso Brasileiro de Informática na Educação (CBIE 2020)

Anais do XXXI Simpósio Brasileiro de Informática na Educação (SBIE 2020)

Tabela 4. Teste de postos sinalizados de Wilcoxon para erros e tempos (em segundos) nos testes de movimento e cliques de mouse $(\alpha=.05)$.

\begin{tabular}{|c|c|c|c|c|c|c|c|c|}
\hline \multirow{2}{*}{ Teste } & \multirow{2}{*}{$\begin{array}{c}\text { Tipo de } \\
\text { Erro }\end{array}$} & \multirow{2}{*}{$\mathbf{N}-\mathbf{N}$} & \multicolumn{3}{|c|}{ Erros } & \multicolumn{3}{|c|}{ Tempos } \\
\hline & & & $Z$ & $p$-valor & $r$ & $Z$ & $p$-valor & $r$ \\
\hline \multirow{3}{*}{$\begin{array}{c}\text { movimento } \\
\text { horizontal }\end{array}$} & tocar & 13-13 & -.845 & .398 & .166 & \multirow{3}{*}{-3.180} & \multirow{3}{*}{$.001^{\star}$} & \multirow{3}{*}{.624} \\
\hline & cruzar & $13-13$ & -2.315 & $.021^{\star}$ & .454 & & & \\
\hline & soltar & 13-13 & -2.943 & $.003^{\star}$ & .577 & & & \\
\hline \multirow{3}{*}{$\begin{array}{c}\text { movimento } \\
\text { diagonal }\end{array}$} & tocar & $13-13$ & -1.267 & .205 & .248 & \multirow{3}{*}{-2.726} & \multirow{3}{*}{$.006^{\star}$} & \multirow{3}{*}{.535} \\
\hline & cruzar & $13-13$ & -.315 & .753 & .062 & & & \\
\hline & soltar & $13-13$ & -1.409 & .159 & .276 & & & \\
\hline \multirow{3}{*}{$\begin{array}{l}\text { movimento } \\
\text { circular }\end{array}$} & tocar & $13-13$ & -.914 & .361 & .179 & \multirow{3}{*}{-2.412} & \multirow{3}{*}{$.016^{\star}$} & \multirow{3}{*}{.473} \\
\hline & cruzar & $13-13$ & -2.804 & $.005^{\star}$ & .550 & & & \\
\hline & soltar & $13-13$ & -1.710 & .087 & .335 & & & \\
\hline \multirow{3}{*}{$\begin{array}{c}\text { movimentos } \\
\text { mistos }\end{array}$} & tocar & 13-13 & -.354 & .723 & .069 & \multirow{3}{*}{-2.517} & \multirow{3}{*}{$.012^{\star}$} & \multirow{3}{*}{.494} \\
\hline & cruzar & $13-13$ & -1.808 & .071 & .355 & & & \\
\hline & soltar & $13-13$ & -.717 & .473 & .141 & & & \\
\hline cliques & não ponto & $13-13$ & -.210 & .834 & .041 & -2.971 & $.003^{\star}$ & .583 \\
\hline
\end{tabular}

\subsection{Avaliação de desempenho com teclado}

O teste de desempenho com teclado também apresentou diferença na média de palavras digitadas com sucesso no pré-treino $\left(\mathrm{N}=13, \bar{x}=9.54, s_{x}=5.238\right)$ e pós-treino $(\mathrm{N}=13$, $\left.\bar{x}=12.23, s_{x}=5.988\right)$. Testes de Shapiro-Wilk e Levene aplicados sobre as amostras indicaram que possuem distribuição normal e variância homogênea ( $p$-valor > .05).

Dessa forma, em uma perspectiva within-subjects, um teste $t$ de Student para amostras pareadas demonstrou diferença estatística significativa ( $\alpha=.05$, bicaudal) $(t=$ $-4.115, p$-valor $=.001)$ e apresentou um coeficiente de Cohen [Pallant 2016] referente ao tamanho de efeito considerado grande $(d=1.141)$.

\subsection{Questões de pesquisa}

(1) Idosos realizam movimentação mais precisa com o mouse após o treinamento com jogos? Sim, mas ela foi pouco expressiva na maioria dos testes. Ainda, os participantes cometeram mais erros após o treinamento em apenas uma situação: erros de "tocar" no teste de movimentos mistos. Como essa situação é bastante atípica, alguns fatores podem fazer parte da causa: ansiedade de concluir o experimento, visto que era o último teste; desenvolvimento de tolerância a pequenos erros em troca de realizar a tarefa com maior velocidade; e aumento de precisão de movimentos que pode ter transformado parte dos erros de "cruzar" as linhas no pré-treino em erros de "tocar" a linha após o treinamento.

(2) Idosos realizam cliques mais precisos com o mouse após o treinamento? A habilidade dos idosos em realizar cliques precisos após o treinamento é indistinguível da habilidade demonstrada antes do treinamento. Esse resultado sugere que talvez seja necessário um tempo de treinamento maior para desenvolver a precisão de cliques ou que os jogos desenvolvidos não tenham sido capazes de treinar adequadamente essa habilidade.

(3) Idosos realizam tarefas mais rapidamente com o mouse após o treinamento? Sim. O treinamento com os jogos propostos aumentou significativamente a ve- 
locidade com que os idosos realizaram os percursos e os cliques nos testes, sugerindo um aumento de desempenho em tarefas que dependam dessas ações. Os elevados tamanhos de efeito indicados na Tabela 4 destacam também a amplitude da melhora de desempenho dos idosos para todos os testes. Assim, constatamos que mesmo um treinamento de poucos dias contribuiu substancialmente para a prática dos idosos com mouse.

(4) Idosos conseguem digitar palavras mais rapidamente usando o teclado após o treinamento? Sim. Os idosos conseguiram digitar mais palavras em um mesmo espaço de tempo após o treinamento, apresentando também um tamanho de efeito muito elevado que demonstra uma melhora bastante expressiva na prática de digitação. Salientamos ainda que, embora as palavras digitadas pelo idoso no pré-teste e no pós-teste fossem as mesmas e estivessem na mesma ordem, é extremamente improvável que tenha havido sua memorização após um evento único, rápido e não marcante de digitação de palavras. A hipótese de melhoria por memorização é ainda mais remota ao considerarmos o intervalo de quatro dias entre os testes de digitação.

\section{Conclusões}

Para garantir a inclusão digital dos idosos, especialmente no que tange ao retorno ao trabalho, é importante que eles aprendam a usar computadores. Sendo mouse e teclado as principais dificuldades no uso do computador, este trabalho teve como objetivo treinar seu uso por meio de jogos, avaliando a efetividade do treino em um experimento.

Nossas principais descobertas incluem que poucas sessões de treinamento (1) contribuem pouco para melhorar a precisão de movimento e clique com o mouse, (2) foram suficientes para aumentar significativamente a velocidade ao usar o mouse, e (3) aumentaram substancialmente a quantidade de palavras digitadas em um intervalo de tempo.

Limitações de nossa pesquisa incluem: (1) tamanho da amostra limitado, (2) diferente frequência dos participantes nos treinamentos, (3) falta de controle em relação a treinamento domiciliar pelos participantes, (4) dificuldade no controle do rigor experimental dos monitores, e (5) possibilidade de interferência no desempenho de movimentação com mouse devido à necessidade de segurar um botão durante a realização dos testes.

Como trabalhos futuros podemos elencar (1) a repetição do experimento com um grupo de controle que realiza treinamento convencional sem jogos, (2) a realização de experimentos com quantidades maiores de sessões de treinamento (duas semanas, um mês, etc.), (3) a obtenção de recurso específico no projeto para custear o deslocamento dos participantes a fim de reduzir a infrequência, e (4) o desenvolvimento de programa específico para testes de desempenho com mouse.

\section{Referências}

Aldrich, C. (2005). Learning by Doing: A Comprehensive Guide to Simulations, Computer Games, and Pedagogy in e-Learning and Other Educational Experiences. JosseyBass Inc., Publishers, San Francisco, CA, EUA.

Bonnechère, B., Sholukha, V., Omelina, L., Van Vooren, M., Jansen, B., and Van Sint Jan, S. (2016). Dynamic balance assessment of elderly patients using serious games. In Proceedings of the 4th Workshop on ICTs for Improving Patients Rehabilitation Research Techniques, REHAB '16, pages 13-16, Nova Iorque, EUA. ACM. 
Brasil (2003). Lei $\mathrm{n}^{\mathrm{o}} 10.741$, de $1^{\circ}$ de outubro de 2003. dispõe sobre o estatuto do idoso e dá outras providências. Diário Oficial [da] República Federativa do Brasil.

Cheng, H.-I., Alifa, R., and Lee, H. (2019). The effectiveness of music therapy system for the elderly with mild cognitive impairment. In Proceedings of the 7th International Conference on Information Technology: IoT and Smart City, ICIT 2019, pages 445448, Nova Iorque, EUA. ACM.

Farias, A. A., Castro, C. A. L., and Almeida, W. R. M. (2015). Desenvolvimento de jogos digitais como estratégia na melhoria de cognição e motricidade de idosos utilizando técnicas de memorização e movimentação. In Proceedings of the Computer on the Beach, pages 81-90, Itajaí, RS, Brasil.

Fiocruz (2020). Covid-19: pesquisa analisa impacto da pandemia no trabalho e renda da pessoa idosa. [Online] https://portal.fiocruz.br/noticia/covid-19-pesquisa-analisaimpacto-da-pandemia-no-trabalho-e-renda-da-pessoa-idosa.

Giacomelli, J., Fernandes, K. C., Lopes, G., Moura, J., Cheiran, J., and Mello, A. (2018). Game design for training of elderly people in mouse and keyboard use. In Proceedings of the XVII Brazilian Symposium on Computer Games and Digital Entertainment, pages 1345-1348, Foz do Iguaçu, Brasil. SBC.

Kachar, V. (2003). Terceira Idade e Informática: Aprender Revelando Potencialidades. Cortez Editora, São Paulo, SP, Brasil.

Neto, H. C. S., Neto, D. P. C., Leite, J. B., Cerejeira, J., and Roque, L. (2016). Cow milking game: Evaluating a serious game for cognitive stimulation with an elderly population. In Proceedings of the International Symposium on Interactive Technology and Ageing Populations, ITAP '16, pages 44-53, Nova Iorque, EUA. ACM.

Niksirat, K., Silpasuwanchai, C., Ren, X., and Wang, Z. (2017). Towards cognitive enhancement of the elderly: A ux study of a multitasking motion video game. In Proceedings of the CHI Conference Extended Abstracts on Human Factors in Computing Systems, CHI EA '17, pages 2017-2024, Nova Iorque, EUA. ACM.

ONU, N. U. B. (2018). Pessoas idosas-onu brasil. [Online] https://nacoesunidas.org/acao/pessoas-idosas/.

Pallant, J. (2016). SPSS Survival Manual. McGraw-Hill Education, Berkshire, England, 6th edition.

Pfeiffer, E. (1975). A short portable mental status questionnaire for the assessment of organic brain deficit in elderly patients. Journal of the American Geriatrics Society, 23(10):433-441.

Pyae, A., Liukkonen, T. N., Saarenpää, T., Luimula, M., Granholm, P., and Smed, J. (2016). When japanese elderly people play a finnish physical exercise game: A usability study. J. Usability Studies, 11(4):131-152.

Vieira, M. C. (2011). O velho e o novo : caminhos para entender a relação dos idosos com as tecnologias digitais. Master's thesis, Faculdade de Educação, Universidade Federal do Rio Grande do Sul, Porto Alegre, RS, Brasil. 